\section{Organoid culture systems: Products supporting one of the biggest revolutions in biological research}

\author{
Ryan Conder, ${ }^{1}$ Leon H. Chew, ${ }^{1}$ \\ Fisal Elstone, ${ }^{1}$ Marianne Lankhorst, ${ }^{1}$ \\ Adam Añonuevo, ${ }^{1}$ Salvatore Simmini, ${ }^{3}$ \\ Wing Chang, ${ }^{3}$ Allen C. Eaves, ${ }^{1,2}$ \\ Terry E. Thomas, ${ }^{1}$ Sharon A. Louis ${ }^{1}$ \\ 1STEMCELL Technologies Inc., \\ Vancouver, Canada; ${ }^{2}$ Terry Fox \\ Laboratory, BC Cancer Agency, \\ Vancouver, Canada; 3STEMCELL \\ Technologies UK Ltd., Cambridge, UK
}

\begin{abstract}
Organoids are stem cell-derived structures that are generated in three-dimensional tissue culture. They are unique since they exhibit a high degree of self-organization and differentiation, and thus recapitulate many of the features of the tissues from which they were derived. Because of this, organoids are now firmly established as an essential tool in medical research, and have the potential to drastically reduce the number of animals required for experimentation.
\end{abstract}

\section{Introduction}

Organoids are stem cell-derived structures that are generated in three-dimensional tissue culture. Organoids are unique since they exhibit an advanced degree of selforganization and differentiation, and thus recapitulate many of the features of the tissues from which they are derived. Because of their similarity to their in vivo counterparts, organoids are emerging as the preferred model system for studying tissue development, homoeostasis, and diseased states in vitro. Organoid technology is widely recognized as one of the greatest technological breakthroughs in basic biological research in the last decade. For these reasons, this technology holds tremendous potential to complement 2D-culture methods and highly reduce the use of animal models in research. STEMCELL Technologies is committed to developing culture media and providing tools that efficiently support the generation and maintenance of multiple organoid culture systems.

\section{Materials and Methods \\ IntestiCult ${ }^{\mathrm{TM}}$ OGM (Mouse)}

Upper intestines of C57BL6/J mice were dissected and washed several times in PBS. The intestinal fragments were then incubated for 20 minutes with Gentle Cell Dissociation Reagent (GCDR) at room temperature (RT) to separate the crypts and villi from the intestinal basal surface. The crypts were then isolated from villi through centrifugation, counted and re-suspended in a 50:50 mixture of Corning ${ }^{\circledR}$ Matrigel ${ }^{\circledR}$ dome and IntestiCult ${ }^{\mathrm{TM}} \mathrm{OGM}$ at 6,000 crypts $/ \mathrm{mL}$. A $50 \mu \mathrm{L}$ droplet of the suspension was gently placed into the center of pre-warmed 12well culture plate wells, creating a dome containing $\sim 300$ crypts/well. The domes were solidified at $37^{\circ} \mathrm{C}$ for $5 \mathrm{~min}$ and the wells were then flooded with $750 \mu \mathrm{L}$ of IntestiCult ${ }^{\mathrm{TM}}$ OGM. Crypts were cultured at $37^{\circ} \mathrm{C}$ for 4-7 days with 3 medium changes per week. After 7 days, organoids were passaged by treating cultures with GCDR for 15 min at RT followed by mechanical disruption into smaller aggregates. The resultant suspension was mixed with IntestiCult ${ }^{\mathrm{TM}} \mathrm{OGM}$ at a 1:6 ratio and then re-plated as above to establish secondary cultures. This protocol was repeated to generate long-term cultures.

\section{IntestiCult ${ }^{\text {TM }}$ OGM (Human)}

Human intestinal crypts were isolated by incubating human small intestine and colon tissue samples with GCDR for 30 minutes at $4{ }^{\circ} \mathrm{C}$ with gentle agitation. The liberated crypts were counted and plated at 1,000 crypts per Corning ${ }^{\circledR}$ Matrigel ${ }^{\circledR}$ dome, flooded with $750 \mu \mathrm{L}$ IntestiCult ${ }^{\mathrm{TM}}$ OGM (Human) and cultured at $37^{\circ} \mathrm{C}$ with 3 medium changes per week. The mature organoids were expanded by harvesting, dissociated by manual agitation and $10 \mathrm{~min}$ GCDR incubation and re-plated at a 1:4 split ratio every $10-12$ days over 15 passages.

\section{STEMdiff' ${ }^{\text {M }}$ Cerebral Organoid Kit}

This kit contains 2 basal media and 5 supplements, which are combined to prepare four separate complete media corresponding to the 4 stages of cerebral organoid formation. Human pluripotent stem cells (hPSCs) maintained in mTeSR1 ${ }^{\mathrm{TM}}$ were dissociated into single cell suspensions, and cultured in Embryoid Body (EB) Formation Medium (days 1 - 5, Stage 1). The resulting EBs were then transferred to Induction Medium (days 6 - 7, Stage 2); next, they were expanded by embedding in Corning ${ }^{\circledR}$ Matrigel ${ }^{\circledR}$ and cultured in Expansion Medium (days 7 - 10,
Correspondence: Salvatore Simmini, STEMCELL Technologies UK Ltd., Cambridge, UK.

E-mail: salvatore.simmini@stemcell.com

Key words: Organoids; IntestiCult; intestine; brain; STEMdiff.

Conference presentation: this paper was presented at the Second Centro 3R Annual Meeting - 3Rs in Italian Universities, 2019, June 20-21, University of Genoa, Italy.

Received for publication: 28 October 2019. Accepted for publication: 6 November 2019.

This work is licensed under a Creative Commons Attribution NonCommercial 4.0 License (CC BY-NC 4.0).

(C) Copyright: the Author(s), 2019

Licensee PAGEPress, Italy

Biomedical Science and Engineering 2019; 3(s2): 102 doi:10.4081/bse.2019.102

Stage 3). The expanded organoids were then cultured in Maturation Medium, with agitation, for extended periods of time (days $10-40+$, Stage 4). Morphological analysis of organoids was performed on days 5, 7, 10 and 40, which are the endpoints of Stages 1 - 4 respectively. Organoids at day 40 were analyzed by RT-qPCR or cryosectioned and processed for immunofluorescence $(>3$ organoids per analysis; 2 human embryonic stem cell (hESC) lines, $\mathrm{n}=2$ and 2 induced pluripotent stem cell (iPSC) lines, $n=2$ ).

\section{Results}

\section{IntestiCult $^{\text {TM }}$ OGM (Mouse)}

The efficiency of mouse intestinal organoids generation in IntestiCult ${ }^{\mathrm{TM}}$ OGM (Mouse) was $64 \pm 8 \%$ (mean $\pm \mathrm{SD}$; $\mathrm{n}=6$ ) by day 5, yielding $\sim 190$ organoids per well. Organoids generally consist of an inner lumen surrounded by a complex arrangement of multiple crypt-like buds. Organoid formation efficiency increased to $86 \pm 4 \%$ $(n=4)$ after the first passage and remained consistently $>85 \%$ over at least 12 passages $(90 \pm 3 \%, n=3)$. Physiological relevance was addressed by demonstrating the presence of intestinal stem cells (Lgr5), polarized enterocytes (Villin), goblet cells (Muc2), enteroendocrine cells (Chg A), and Paneth cells (Lysozyme) by immunocytochemistry and qPCR analysis of organoids at each passage.

\section{IntestiCult ${ }^{\mathrm{TM}}$ OGM (Human)}

Spherical human intestinal organoid structures could be identified within 2 days of initial seeding. Organoids analyzed by 
immunohistochemical and qRT-PCR analyses for intestinal stem (LGR5 and AXIN2), Paneth (LYZ), enteroendocrine (CHGA), goblet (MUC2) and enterocyte (VIL1) cell marker expression, revealed that organoids were comprised of both stem and differentiated cell types, demonstrating cultured human organoids closely resembled human intestine structure. Organoids expanded for $>1$ year in culture with an average passaging ratio of $1: 6$ every 8 to 12 days.

STEMdiff'M Cerebral Organoid Kit

The efficiency of the STEMdiff ${ }^{\mathrm{TM}}$ Cerebral Organoid Kit was tested across multiple cell lines $(2 \mathrm{hESCs}, \mathrm{n}=2$ and 2
iPSCs, $\mathrm{n}=2)$ for EB generation ( $100 \%$ success, $n=128 / 128$ ), expansion ( $>95 \%$ exhibited extensive folding of neuroepithelia, $\mathrm{n}=$ $104 / 107)$ and maturation $(>60 \%$ of organoids were $>1 \mathrm{~mm}$ in diameter with dense cores, $\mathrm{n}=62 / 94$ ). In vivo, the human cortex consists of progenitor and neuronal populations that organize into distinct layers. The mature organoids exhibited a similar architecture with neural progenitors $\left(\mathrm{SOX}_{2}{ }^{+}, \mathrm{PAX}^{+}\right)$localized in apical regions surrounding a central ventricle. Adjacent to the apical progenitors, neuronal progenitors (TBR2 ${ }^{+}, \mathrm{Ki}^{-67^{+}}$) were found abutting neurons $\left(\mathrm{CTIP} 2^{+}, \mathrm{MAP}^{+}, \mathrm{TBR} 1^{+}\right)$, resembling the intermediate zone and cortical plate regions.

\section{Conclusions}

STEMCELL Technologies supports the organoid culture system revolution in biological research by developing products like IntestiCult ${ }^{\mathrm{TM}}$ OGM (Mouse and Human) and STEMdiff ${ }^{\mathrm{TM}}$ Cerebral Organoid Kit that enable generation of organoids in a highly reproducible manner, thus reducing experimental variability and increasing accuracy of results for researchers to advance their scientific discoveries. 